\title{
Optimization study on Niosome Loaded Ficus Deltodia Extract.
}

\author{
Noorazwani Zainol ${ }^{1}$, Chen Siew Chin, ${ }^{2}$ Nor Rashidah Ahmed ${ }^{1}$, Noorazwani Zainol ${ }^{1}$, Azila Abdul Aziz ${ }^{2}$, Rahimah Sabtu ${ }^{1}$ \\ Harisun Yaakob ${ }^{2 *}$ \\ ${ }^{1}$ Food and Nutraceutical Unit, Institute of Bioproduct Development, Universiti Teknologi Malaysia, 81310, Johor Bahru, Johor, \\ Malaysia \\ ${ }^{2}$ Department of Bioprocess Engineering, Faculty of Chemical and Energy Engineering, Universiti Teknologi Malaysia, 81310, Johor, \\ Malaysia.
}

\begin{abstract}
Ficus deltoidea (FD) plant extract is not able to cross the lipidic biomembranes of the skin as they are hydrophilic in nature. In order to overcome this problem FD were encapsulated with noisome, a novel lipid delivery system that potentially increases the absorption. This research study on optimum formulation and process condition of niosome loaded FD extract. The factors involved in formulation were amount of Span 60, amount of cholesterol and amount of Labrasol. Their corresponding responses for the design were entrapment efficiency (EE), particle size (PS) and zeta potential (ZP). The optimum formulation obtained were $0.6 \mathrm{mmol}$ of Span $60,0.4 \mathrm{of} \mathrm{mmol}$ Cholesterol, and $0.1 \mathrm{mmol}$ of Labrasol. Results obtained showed improved activity on skin penetration compared to free FD.
\end{abstract}

\section{INTRODUCTION}

In recent years, natural herbs are widely use in cosmeceutical area due to their fewer side effects as compared to the synthetic or chemical based active ingredient products. Consumer awareness towards hazardous side effects of those chemical derived cosmetic products highly increase the demand of herbs based cosmetic. However, there are several common problems to have herbs applied in cosmeceutical field, as several phytoconstituents (such as flavonoids, terpenoids, tannins etc.) have poor absorption through skin. This is due to their poor lipid/water solubility and/or incompatible molecular size [1]. Benefits of Ficus deltoidea extract not only on promoting health effect but also giving a beneficial effect to the skin such as antiaging and whitening effect [2]. However Ficus deltoidea is mainly hydrophilic in nature, which may reduce their absorption through skin as they not able to cross the lipidic biomembrane [1]. There are some methods were reported to overcome phytochemicals bioavailability problems such as purification and isolation, however this kind of method will reduce the efficacy of herbs due to the loss of synergy effect. Therefore, a good delivery vehicle is needed to deliver the extracts to targeted site and increase the using bioavailability. There were only few herbs being reported whereby niosome as a delivery vehicle such as Ginkgo Biloba, Curcuma longa linn, and Silybum marianum. Coacervation and phase separation, a method for proniosomes preparation is chosen for preparation of niosomal dispersion among other methods as this is a simple practical method for the routine and large scale production with the minimum use of organic solvent [3]

In this study, optimum formulation for DF loaded niosomes was investigated. Design of experiment (DOE) was carried out to deal with the parameters that affect niosomes production. The niosomes were characterized based on encapsulation efficiency, particle size, zeta potential and skin penetration.

\section{MATERIALS AND METHODS}

Tween 20, Tween 60, Span 20, Span 60, Span 80, Sephadex G-50 (medium), monobasic sodium phosphate, and dibasic sodium phosphate were purchased from Sigma Aldrich (St. Louis, MO, USA). Absolute ethanol $(99.9 \%)$ and 1-propanol was purchased from Qrec (Chonburi, Thailand). Labrasol was purchased from Gattefosse Corporation (Saint-Priest, France).

Dried leaves FD purchased from Malaysian Agriculture and Research Development Institute (MARDI), Muadzam, Pahang. The dried leaves were dried further for 2 days at $45^{\circ} \mathrm{C}$ before being ground to a powder, which was extracted with boiling water for $1 \mathrm{~h}$, after which the infusion was filtered and the filtrate was spray-dried to form a powder [4]. $1 \mathrm{~g}$ of obtained powdered FD extract was dissolved in $50 \mathrm{~mL}$ of water and centrifuged at 10000 g-force for $5 \mathrm{~min}$, the supernatant was then filtered with nylon membrane filter (Agilent techologies) with $0.2 \mu \mathrm{m}$ pore size, the resultant filtrate was acts as nonsolvent for span 60 in niosomes preparation.

\subsection{Preparation of Niosome}

Niosome with FD extract encapsulated were prepared by coacervation phase separation method and followed by hydration as described by [5]. To study the effect of cholesterol, Labrasol, and surfactant on niosomes formation, first of all, Span 60 was mixed with an appropriate amount of lipid (Labrasol and cholesterol) in a $10 \mathrm{~mL}$ glass vial. $1 \mathrm{~mL}$ of solvent (absolute ethanol) was then added to the mixtures and the vial was tightly sealed before putting in a water bath at $60^{\circ} \mathrm{C}$ while shaking until

* Corresponding author: harisun@ibd.utm.my 
complete dissolution of the mixture. The specified amount of FD extract (nonsolvent) was then added to the mixture while warming in the water bath for 3-5 min till clear or translucent solution obtained. Niosomal suspension was then obtained by hydration of $200 \mathrm{~mL}$ of distilled water for $10 \mathrm{~min}$ in water bath. The obtained niosomal suspensions were finally proceed with downsizing with a high-pressure homogenizer (Microfluidizer M-110P, Newton, MA, USA) with constants parameter (750 bar and 4 cycles). The encapsulation efficiency and niosome mean particle size were determined.

\subsection{Characterization of Niosomes}

Niosome were characterized based on their entrapment efficiency (EE), particle size (PS), zeta potential (ZP) and penetration study.

\subsubsection{Entrapment efficiency (EE)}

The EE was determined by minicolumn centrifugation method [6]. The column (EconoSpin size exclusion columns, Epoch life science, USA) were prepacked hydrated sephadex G-50 which come with inert support as shown in Figure 3.2. These columns were then placed in the microcentrifuge tubes and the whole assembly centrifuged to remove excess water. Niosomes suspension was then applied to the dried bed and the assembly centrifuged at $1700 \mathrm{rpm}$ for 2 minutes. The niosomes were expelled in the void volume, on the other hand the excess unentrapped extract was trapped in the void volume. The expelled niosomes which collected in the microcentrifuge tubes were disrupted with $50 \%$ n-propanol with ratio $1: 1$ in an ultrasonic bath for 20 minutes. The resultant mixture was filtered with $0.2 \mu \mathrm{m}$ syringe nylon filter and the filtrate was subjected to total phenolic content assay. The entrapment efficiency can be represented as following Equation 1.0:

$$
\mathrm{EE} \%=
$$

total phenolic content inside the vesicles $\times 100$ total phenolic content added

\subsubsection{Measurement of Particle Size}

For the niosomal suspension, vesicular size and distribution will be determined by Zetasizer (Malvern Zetasizer Nano ZS, Malvern Instruments, Worcestershire, UK). The analyses were performed by dynamic light scattering (DLS) principle at constant temperature $25^{\circ} \mathrm{C}$, material refractive index at 1.45 and absorption index 0.001. Each sample was measured three times and results are expressed as mean \pm standard deviation. The polydispersity index (PDI) was used as a measure of unimodal size distribution. A small value of PDI (0.1) indicates a homogenous population, while a PDI. 0.3 indicates a higher heterogeneity.

\subsubsection{Zeta potential determination}

Zetasizer (Malvern Zetasizer Nano ZS, Malvern Instruments, Worcestershire, UK) was used to measure the zeta potential of niosomes with Electrophoretic Light
Scattering principle. Zeta potential was determined 3 times for each sample, and results were automatically calculated by the analyzer using the Smoluchowski equation.

\subsubsection{Skin penetration study}

This study was performed by a transdermal Franz diffusion system (PermGear, Germany) that composed of six horizontal diffusion cells (effective area $=1.07 \mathrm{~cm}^{2}$ ), a thermally control circulating water bath and a magnetic stirrer for each of cells. The experiments were conducted by using the abdnominal skin of 9 weeks old rats [7]. The shaved skin was cut into squares and the subcutaneous fat was carefully removed, and the skin was pre-equlibrated in Phosphate Buffer Solution (PBS, $\mathrm{pH}=6.5$ ) at $4^{\circ} \mathrm{C}$ for 24 hour.

The skins were mounted on the receptor compartment with the stratum corneum side facing the donor cell. The receiver compartments $(3.7 \mathrm{~mL})$ were filled with PBS solution $(\mathrm{pH} 6.5)$ and the donor compartments are filled with $2.7 \mathrm{~mL}$ of the same PBS solution. The diffusion calls were equilibrated by the re-circulating water bath at $37 \pm$ $1{ }^{\circ} \mathrm{C}$, provides a skin surface temperature of $32 \pm 1^{\circ} \mathrm{C}$ (Misik et al., 2011). The fluid in the donor and receptor compartment was stirred continuously by magnetic stirrer. Setup was run for half an hour to allow the equilibrium of temperature. $1 \mathrm{~mL}$ of samples was then pipette into the donor compartment.

Sampling was done at $0.5,1,1.5,2.0,3,4,5,6,8,10,14$, 18 and $24 \mathrm{~h}$. At each point, $1 \mathrm{~mL}$ of aliquots was withdrawn from the receiver compartment and thereafter, $1 \mathrm{~mL}$ of PBS solution was added as replacement to the receiver compartment. The collected samples were disrupted with $50 \%$ n-propanol with ratio $1: 1$ in an ultrasonic bath for 20 minutes. The resultant mixture was filtered with $0.2 \mu \mathrm{m}$ syringe nylon filter and the filtrate was subjected to total phenolic content assay in order to obtain the concentration of extract.

Flux $\left(\mu \mathrm{g} / \mathrm{cm}^{2} \mathrm{~h}\right)$, were calculated using the linear regression interpolation of the experimental data at a steady state using Fick's Law [7][8]

\section{Result and Discussion}

\subsection{Formulation Optimization}

Formulation optimization involved three parameters which were the amount of cholesterol (A), Span 60 (B) and labrasol (C). The lower and upper limits of parameters were determined from the previous studies (unpublish data). Table 1 presents the DOE for FD niosomes where their responses were particle size (PS) (Y1), zeta potential $(\mathrm{ZP})(\mathrm{Y} 2)$ and encapsulation eficiency $(\mathrm{EE})(\mathrm{Y} 3)$. 


\begin{tabular}{|l|l|l|l|l|l|l|}
\hline Modified & 0.40 & 0.60 & 0.10 & $\begin{array}{l}151 . \\
20\end{array}$ & $\begin{array}{l}49.7 \\
0\end{array}$ & 78.43 \\
\hline
\end{tabular}

Table 1. DOE for FD Niosomes

\begin{tabular}{|c|c|c|c|c|c|c|}
\hline Run & $\begin{array}{c}\text { A } \\
(\mathrm{mmol})\end{array}$ & $\begin{array}{c}\text { B } \\
(\mathrm{mmol})\end{array}$ & $\begin{array}{c}\mathrm{C} \\
(\mathrm{mmol})\end{array}$ & $\begin{array}{c}\text { Y1 } \\
(\mathrm{nm})\end{array}$ & $\begin{array}{c}\text { Y2 } \\
(-\mathrm{mV})\end{array}$ & $\begin{array}{c}\text { Y3 } \\
(\%)\end{array}$ \\
\hline 1 & 0.29 & 0.62 & 0.09 & 203.267 & 45.2 & 67.3 \\
\hline 2 & 0 & 1 & 0 & 232.9 & 50.13 & 76.9 \\
\hline 3 & 0.18 & 0.64 & 0.18 & 176.2 & 45.267 & 84.63 \\
\hline 4 & 0 & 0.8 & 0.2 & 269.2 & 47.7 & 51.8 \\
\hline 5 & 0.09 & 0.82 & 0.09 & 283.5 & 50.3 & 84.13 \\
\hline 6 & 0.4 & 0.6 & 0 & 198 & 49.233 & 78.57 \\
\hline 7 & 0.09 & 0.62 & 0.29 & 300 & 40.76 & 61.1 \\
\hline 8 & 0 & 0.6 & 0.4 & 335.967 & 33 & 35.8 \\
\hline 9 & 0.25 & 0.5 & 0.25 & 180.13 & 42.4 & 75.3 \\
\hline 10 & 0 & 0.6 & 0.4 & 335.8333 & 42.467 & 54.3 \\
\hline 11 & 0 & 1 & 0 & 224.633 & 52.2 & 66.67 \\
\hline 12 & 0.2 & 0.8 & 0 & 191.767 & 48.267 & 87.9 \\
\hline 13 & 0.25 & 0.5 & 0.25 & 176.2 & 44.7 & 87.3 \\
\hline 14 & 0.4 & 0.6 & 0 & 183.867 & 51.3 & 67.3 \\
\hline
\end{tabular}

\subsection{Effets of Formulation Ingrédients and their interactions on Réponses}

Figure 1 shows the three-dimensional plot representing the effect of the interaction of variables on PS for FD niosomes. Based on the contour plot, particle size decreased while cholesterol (X1) increased, or when labrasol decreased. Therefore the minimum vesicle size can be achieved at a region where high amount of cholesterol and Span 60, low amount labrasol. Figure 2 shows the three-dimensional plot represents the effect of the interaction of variables on ZP. Based on the contour plot, the maximum particle size can be achieved at high amount of cholesterol and Span 60, low amount labrasol. Effect of mixture component towards EE could be observed through Figure 3, maximum EE could be obtained at a region where high cholesterol and low labrasol, however when increment of cholesterol exceed 30 mole \%, EE started to decreased as existence of cholesterol may compete with the drug for packing space [9]. The response that significantly affected the mixture component for niosomes production was PS, ZP and EE. The optimum value for the parameter were shown in Table 2, with the difference is not more than $15 \%$.

Table 2. Check point analysis

\begin{tabular}{|l|l|l|l|l|l|l|}
\hline \multirow{2}{*}{$\begin{array}{l}\text { Check } \\
\text { point }\end{array}$} & \multicolumn{4}{|l|}{ Processing parameters } & \multicolumn{2}{l|}{ Output } \\
\cline { 2 - 7 } & $\begin{array}{l}\text { Cholest } \\
\text { erol } \\
(\mathrm{mmol})\end{array}$ & $\begin{array}{l}\text { Span } \\
60 \\
(\mathrm{~mm} \\
\mathrm{ol})\end{array}$ & $\begin{array}{l}\text { Labras } \\
\text { ol } \\
(\mathrm{mmol})\end{array}$ & $\begin{array}{l}\text { PS } \\
(\mathrm{nm})\end{array}$ & $\begin{array}{l}\text { ZP } \\
(- \\
\mathrm{mV})\end{array}$ & $\begin{array}{l}\text { EE } \\
(\%)\end{array}$ \\
\hline Optimum & 0.37 & 0.57 & 0.07 & $\begin{array}{l}176 . \\
2\end{array}$ & $\begin{array}{l}47.8 \\
329\end{array}$ & $\begin{array}{l}74.22 \\
05\end{array}$ \\
\hline
\end{tabular}

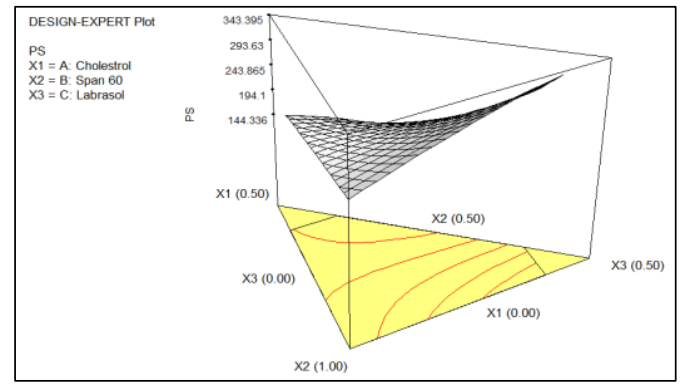

Figure 1. 3-dimensional response surface plot representing the effect of the interaction of variables on PS

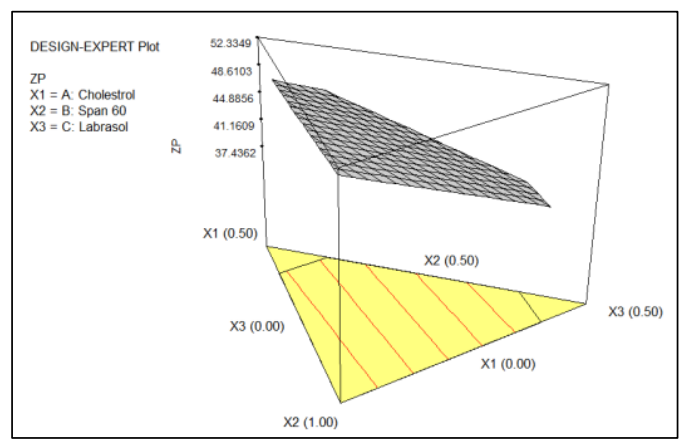

Figure 2. 3-dimensional response surface plot representing the effect of the interaction of variables on $\mathrm{ZP}$

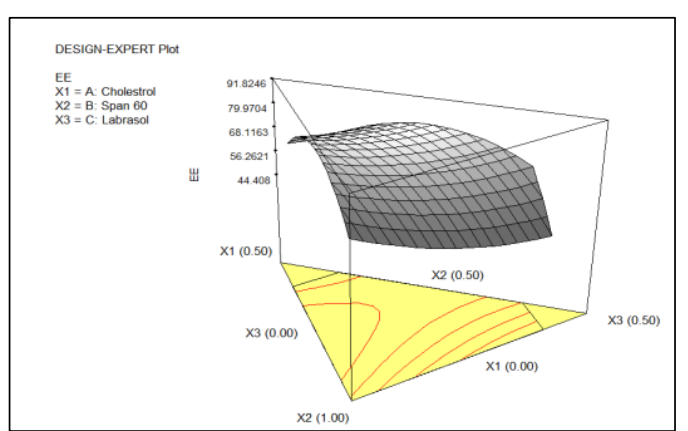

Figure 3. 3-dimensional response surface plot representing the effect of the interaction of variables on $\mathrm{EE}$

\subsection{Penetration of FD Niosomes in Rat Skin}

Penetration study was done using Franz horizontal diffusion cells with rat skin used as the barier. The FD niosomes were obtained using the optimized formulation of $6: 1: 4$ of Span 60 :labrasol :cholesterol. The permeations of free FD extracts and FD niosomes through rat skin were compared. Table 3 showed that the FD niosomes diffuse faster than FD extract without any encapsulation. Higher values of flux (1.56 times) obtained 
with FD niosomes $0.067 \mu \mathrm{g} / \mathrm{cm}^{2} \mathrm{~h}$ than that obtained with FD extract only $0.043 \mu \mathrm{g} / \mathrm{cm}^{2} \mathrm{~h}$. Besides, deposition of extract by FD niosomes $0.157 \mu \mathrm{g}$ also higher than FD extracts $0.124 \mu \mathrm{g}$. Fig 4 shows the trend of culmulative permeation of free FD extracts and FD niosomes through lab skin for the period of 24 hours and permeation coefficient through rat skin, which were calculated and tabulated in Table 3.

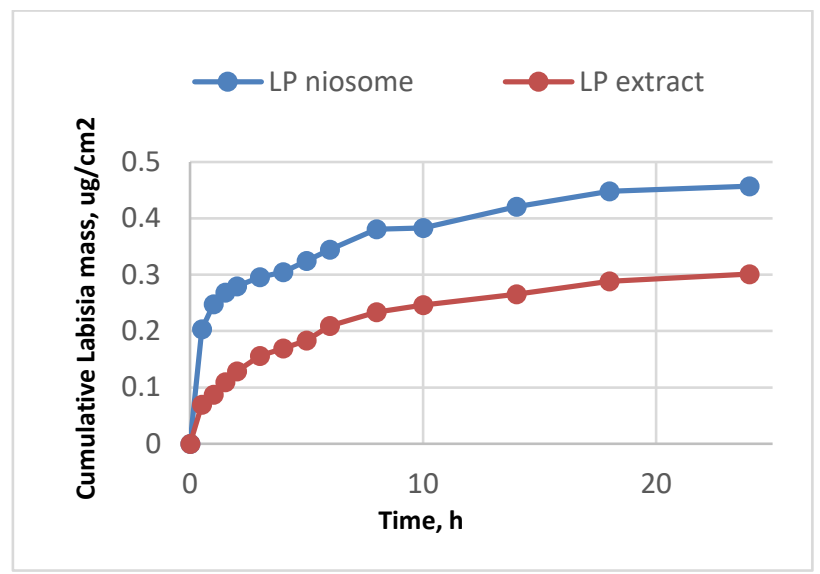

Figure 4. Trend of cumulative permeation through rat skin for the period of 24 hours.

Table 3. Comparison of transport parameters for penetration and the amount of active ingredients in the skin

\begin{tabular}{|l|l|l|l|l|}
\hline Sample & $\begin{array}{l}\text { Cumulative } \\
\text { Permeation } \\
Q_{\mathrm{t}} / \mathrm{S} \\
\left(\mu \mathrm{g} / \mathrm{cm}^{2}\right)\end{array}$ & $\begin{array}{l}\mathrm{J}_{\mathrm{ss}}, \\
\mathrm{h})\end{array}$ & $\begin{array}{l}\mathrm{Kp} / \mathrm{cm}^{2} \\
10^{-1} \\
\mathrm{~cm} / \mathrm{h})\end{array}$ & $\begin{array}{l}\text { Amount of } \\
\text { active } \\
\text { ingredients } \\
\text { in the skin, } \\
\mu \mathrm{g}\end{array}$ \\
\hline $\begin{array}{l}\text { FD extract } \\
\text { without } \\
\text { encapsulat } \\
\text { ion (FDe) }\end{array}$ & $0.212 \pm 0.02$ & 0.0430 & 0.72 & $0.124 \pm 0.027$ \\
\hline $\begin{array}{l}\text { FD } \\
\text { niosomes }\end{array}$ & $0.234 \pm 0.34$ & 0.0672 & 1.12 & $0.157 \pm 0.012$ \\
\hline
\end{tabular}

\section{Conclusion}

The optimum formulation obtained for FD loaded niosomes was Span 60, labrasol and cholesterol in the ratio of $6: 1: 4$. The optimized niosomes showed the feasibility to be applied in cosmetic formulation.

\section{Acknowledgement}

The authors would like to thank the Ministry of Education Malaysia, Institute of Bioproduct Development, Universiti Teknologi Malaysia and research vote 4J271 for financial assistance to this research study.

\section{Referrences}

1. Manach, C., Scalbert, A., Morand, C., Rémésy, C. and Jiménez, L. (2004). Polyphenols: food sources and bioavailability. The American Journal of Clinical Nutrition. 79(5), 727-47.

2. Oh, M.-J., Abdul Hamid, M., Ngadiran, S. and Seo, Y.-K. (2010). Ficus deltoidea (Mas cotek) extract exerted antimelanogenic activity by preventing tyrosinase activity in vitro and by suppressing tyrosinase gene expression in B16F1 melanoma cells. Arch Dermatol Res.

3. Fang, J., Hong, C., Chiu, W. and Wang, Y. (2001). Effect of liposomes and niosomes on skin permeation of enoxacin. International Journal of Pharmaceutics. 219, 61-2.

4. Sulaiman, M.R., Hussain, M.K., Zakaria, Z.A., Somchit, M.N. and Moin, S. (2008). Evaluation of the antinociceptive activity of Ficus deltoidea aqueous extract. Fitoterapia. 79, 557-561.

5. Vora, B., Khopade, A. and Jain, N. (1998). Proniosome based transdermal delivery of levonorgestrel for effective contraception. Journal of controlled release. 54, 149-165.

6. Fry, D., White, J. and Goldman, I. (1978). Rapid separation of low molecular weight solutes from liposomes without dilution. Analytical biochemistry. 90(2), 809-815.

7. Shah, K. a, Date, A. a, Joshi, M.D. and Patravale, V.B. (2007). Solid lipid nanoparticles (SLN) of tretinoin: potential in topical delivery. International journal of pharmaceutics. 345(1-2), 163-71.

8. Sintov, A.C. and Shapiro, L. (2004). New microemulsion vehicle facilitates percutaneous penetration in vitro and cutaneous drug bioavailability in vivo. Journal of controlled release: official journal of the Controlled Release Society. 95(2), 173-183.

9. Balakrishnan, P., Shanmugam, S., Lee, W.S. and Lee, W.M. (2009). Formulation and in vitro assessment of minoxidil niosomes for enhanced skin delivery. International Journal of Pharmaceutics. 377(1), 1-8. 